\title{
Relationships between body size and abundance in ecology
}

Ethan P. White ${ }^{1,2}$, S.K. Morgan Ernest ${ }^{2}$, Andrew J. Kerkhoff ${ }^{3}$ and Brian J. Enquist ${ }^{1}$

${ }^{1}$ Department of Biology, Utah State University, Logan, UT 84322, USA

${ }^{2}$ Department of Ecology and Evolutionary Biology, University of Arizona, Tucson, AZ 85721, USA

${ }^{3}$ Department of Biology and Department of Mathematics, Kenyon College, Gambier, OH 43022, USA

Corresponding author: White, E.P. (epwhite@biology.usu.edu). 
Body size is perhaps the most fundamental property of an organism and is related to many biological traits, including abundance. The relationship between abundance and body size has received much attention, both to quantify the form of the relationship and to understand the processes that generate it. However, progress is impeded by the underappreciated fact that there are four distinct, but inter-related, relationships between size and abundance that are often confused in the literature. Here, we review and distinguish between these four patterns, and discuss the linkages between them. We argue that a synthetic understanding of size and abundance relationships will result from more detailed analysis of individual patterns and from careful consideration of how and why the patterns are related to one another.

\section{Introduction}

Body size is one of the most fundamental properties of an organism. Because body size is related to lifespan, home range size and other aspects of life history and ecology [1,2], the relationship between body size and abundance is an essential link between the individual- and populationlevel traits of species and the structure and dynamics of ecological communities [3-5]. In addition, because body size is one of the primary determinants of metabolism and, therefore resource use [1,2], the relationship between size and abundance also reveals how resources are partitioned in ecological systems.

While the importance of size-abundance relationships has been widely recognized in both terrestrial and aquatic ecology (e.g. Refs $[3,6,7])$, controversy has arisen regarding the form of the relationship and its implications for ecology and evolution (e.g. Refs [8-10]). In part, the controversy is rooted in the underappreciated fact that there are four distinct relationships 
between body size and abundance, which are routinely confused with one another in the literature (see Glossary; Box 1; see also Ref [11]). Because each of these relationships is generated by different combinations of processes, they must be evaluated separately. With the goal of better understanding how and why abundance and size are related, here we clarify the four separate relationships and propose standard terminology to reduce confusion; review the empirical patterns demonstrated for each relationship; discuss the processes that might generate the different patterns, and the possible linkages between them; and propose novel directions for future size-abundance research.

\section{The four size-abundance patterns}

The global size-density relationship

The global size-density relationship (GSDR) is one of the most studied of the size-abundance relationships, but it is also one of the hardest to interpret. For large compilations of population densities, the relationship between a species average mass $\left(M_{s p}\right)$ and its average density ( $N_{\text {comp }}$ ) is generally well fit by a power function $\left(r^{2} \mathrm{~s}>80 \%\right)$ with an exponent near -0.75 so that $N_{\text {comp }}=c M_{s p}^{-3 / 4}$ (also known as Damuth’s Rule; Figure 1a) [12-15]. Although such a regular pattern suggests a simple explanation, the way GSDRs are constructed presents a significant challenge for interpreting the processes they reflect. The datasets for GSDRs are conglomerates of species' abundances aggregated at the continental or global scale, with abundances for each species taken from locations that happen to be reported in the literature. As a result, many of the species do not coexist at the reported densities. These densities may also be "biased toward maximum population densities" because "ecologists tend to study populations where they are abundant” [16] (see also [8,10,17]). 
The Damuth's Rule form of the GSDR is particularly interesting, because the scaling of abundance with body size ( $N_{\text {comp }} \propto M_{s p}^{-3 / 4}$ ) is approximately inversely proportional to the scaling of metabolic rate ( $B \propto M_{s p}^{3 / 4}$ ). As a result, Damuth’s Rule suggests that population energy use $\left(B \times N_{\text {comp }}\right.$ ) is approximately invariant with respect to mass (as $M_{s p}^{3 / 4} \times M_{s p}^{-3 / 4}=M_{s p}^{0}$ ) [18]. This so-called "energetic equivalence rule” (EER) fascinates ecologists because it suggests that some combination of physiological and ecological processes results in energetic tradeoffs, such that resources are divided equally across species populations, regardless of their body size (but see Box 2).

Another clue that energetic processes might underlie the power-law form of the GSDR comes from the differences in coefficients (i.e., $c$ ) that exist between groups (e.g. endotherms versus ectotherms). Most notably, shifts in the coefficients are approximately proportional to the efficiency of energy transfer between trophic levels [19-21] and are inversely proportional to temperature-driven shifts in metabolic rate [12,13]. Thus, differences in $c$ among clades or functional groups may result from the combined effects of energy availability in the environment and the rate at which individuals use energy [19-21]. For example, when resource availability is quantitatively factored into the GSDR, the variation around the relationship is reduced for mammalian carnivores [22]. However, it is not clear how resource availability and division, which are by definition local-scale processes, can be invoked to explain a global-scale pattern of abundance and body size $[16,23,24]$. At the very least, such an explanation must assume that energy availability is uncorrelated with size across the continental to global domain of the GSDR. Furthermore, even within taxonomic groups, species of different size often consume different types of resources. As such, it seems unlikely that a simple energetic or resource-based model is entirely sufficient to explain the GSDR [16,23,24]. 
In truth, we lack a complete understanding of why the power law form of the GSDR emerges at large spatiotemporal scales when average (or possibly maximum) population densities are considered $[10,24]$. Given the scale at which the GSDR pattern is evident, it seems likely that the underlying processes are not strictly ecological, but that an evolutionary component also plays an important role $[9,13,25]$. However, there have been few attempts to understand the EER from an evolutionary perspective. One recent evolutionary model produced power law size density relationships (SDRs), but the exponents were often more negative than 0.75 [9]. However, the model was based on localized interactions among species generating local, rather than global, SDRs (see below). More recently, Damuth [26] has shown that the EER can emerge from the evolutionary consequences of competitive processes and zero-sum dynamics (i.e., Van Valen’s Red Queen; [27]). Continued work on models that incorporate evolutionary processes should provide additional insight into the mechanisms underlying Damuth's Rule, the EER and the GSDR in general.

\section{Local size-density relationships}

The GSDR was originally interpreted as also applying directly to local communities [18]. However, similar analyses conducted at local scales (Local size-density relationships; LSDRs) often show either weak power law relationships with exponents significantly shallower than 0.75 (average exponent -0.25 [28]; Figure 1b) or constraint triangles, in which density varies widely beneath an upper bound that either decreases monotonically with body size or has an intermediate maximum $[7,10,16,17]$. In general for LSDRs body size explains little variation in abundance; $r^{2}<15 \%$ in contrast to $r^{2}>80 \%$ for GSDRs [28], although LSDRs tend to be stronger in aquatic systems $[14,23,29-31]$. The observed deviations from energetic equivalence in the LSDR are suggestive of size biases in resource acquisition that could be driven by size 
asymmetries in competition (e.g., [32-34]), or could result from differences in the availability of resources to different size species (e.g., [35,36]).

However, in order to fully understand the implications of empirical LSDRs for processes of community assembly it is necessary to understand why LSDRs and GSDRs differ. The prevailing viewpoint holds that the GSDR probably represents only the maximum upper boundary on local abundance [6,17], reflecting large scale processes rather than local scale division of resources within a community (Figure 2) [23]. It is well established that a species will generally be highly abundant only in some small portion of its range and rare elsewhere $[7,37,38]$. As a result, local assemblages are likely to include species with populations that are below the densities used to construct the GSDR, especially if the GSDR is biased towards maximum densities [7,17]. This would result in Damuth’s Rule ( $N \propto M_{s p}^{-3 / 4}$ ) holding locally only as a upper bound, but exploration of this possibility has produced equivocal results [8,16,17].

An alternative hypothesis posits that LSDRs differ from GSDRs because LSDRs typically examine a smaller range of body sizes $[7,39,40]$. Observing a small portion of the overall relationship necessarily accentuates the noise in the local sample [39]. This hypothesis could explain why aquatic LSDRs are stronger than terrestrial ones, because aquatic sampling methods typically capture a much greater proportion of the global size range than terrestrial studies.

It should be straightforward to distinguish between these two hypotheses by overlaying GSDRs with equivalent LSDRs. The first hypothesis suggests that most of the LSDR should fall below the GSDR, with only the upper boundary overlapping the global densities. In contrast, the second hypothesis suggests that the LSDR should fall entirely within the GSDR. However, to our 
knowledge no comprehensive evaluation of these predictions has been conducted [39]; leaving the relationship between GSDRs and LSDRs unclear (Figure 2).

The individual size distribution or size spectrum

Whereas SDRs have been the primary focus of size-abundance studies in terrestrial ecology, aquatic ecologists mainly study the individual size distribution (ISD; often referred to in the aquatic literature as the size spectrum). The ISD is the frequency distribution of individual body sizes in a community, irrespective of species. Thus, the $n_{i}$ individuals in the $i$ th body size class of average mass $\overline{m_{i}}$ can represent one or more species, and each species can occur in one or more size classes. Because the ISD studies individuals instead of species, it focuses on the question: how are resources divided across body sizes?

Three major classes of patterns have been observed for individual size distributions: (i) monotonically decreasing, where the smallest size classes contain the most individuals and the largest size classes the fewest; these patterns have typically been characterized by power functions [3]; (ii) unimodal, where some intermediate size class contains the most individuals [41,42]; and (iii) multimodal, where the distribution is characterized by multiple peaks $[43,44]$ (Figure 1c).

Aquatic research has focused on ISDs both within and across trophic levels, although aquatic researchers typically study the distribution of biomass spectra across size classes instead of abundance (Box 3). Within trophic levels, the relationship is sometimes considered to be concave under log-log transformation and sometimes a simple power law (see Ref [3] for a detailed review). Across trophic levels, abundance is generally related to body size (measured as volume; Box 3) by a decreasing power law. Such aquatic size distributions are remarkably 
consistent across ecosystems, and they are well modeled using predator:prey size ratios, constraints on trophic efficiency, and allometric relationships for physiological processes [3,45].

In general, less is known about the ISD in terrestrial systems [3]. The one exception is size distributions in tree communities, which are often studied using demographic models [46]. Originally, tree ISDs were characterized as either negative exponentials or power laws $[47,48]$. However, recent work suggests these distributions may deviate from these simple models, especially in the largest trees [46,49]. In contrast to trees, ISDs are rarely studied in terrestrial animals. The few studies available exhibit all three major classes of pattern. Insects have shown monotonic declines in abundance with size [50], unimodal relationships with intermediate sizes having the maximum abundance [41], and complex multimodal distributions [44]. One study of bird communities showed mostly multimodal distributions [44], and two recent studies are suggestive of similar patterns in mammal communities [36,51].

The general variability and complexity of ISDs across groups make it hard to draw general conclusions about these relationships. However, important differences between aquatic and terrestrial ecosystems and between plants and animals could provide insights into the processes structuring communities. For example, in aquatic systems, the relationship between predator and prey size is more constrained than that in terrestrial systems (e.g. Refs [3,52]), particularly when considering trophic links to primary producers. Perhaps this difference contributes to the complexity of ISDs in terrestrial systems. Another possible factor affecting the form of the size distribution is parental care. Parental care allows juveniles to avoid competition with smaller species. In aquatic and tree communities, juveniles of a large species are not always shielded in this way and might have to compete with adults of small species. Differences between aquatic and terrestrial systems make it unlikely that a single model can be directly 
applied to both. However, it is probably worthwhile for terrestrial ecologists to integrate components from aquatic models (i.e. size-related resource availability and use, predator-prey relationships, and physiological allometries) with other processes known to be important for size structure in terrestrial systems (e.g. competition $[36,53,54]$ and habitat structure $[35,55])$.

\section{Cross-community scaling relationship}

The most well known form of the cross-community scaling relationship (CCSR) is the selfthinning rule for plant $[56,57]$ and intertidal [58] communities. Self-thinning tends to be studied in sessile communities, where, as organisms grow, there is space for fewer and fewer individuals.

This leads to a negative relationship between mean organism size, $\overline{m_{\text {ind }}}$, and total community abundance, $n_{\text {tot }}$. Analyses are conducted either by tracking a community through time or by comparing different communities across space. Communities in self-thinning studies are often monospecific stands, so that the assemblage is actually a single-species population. In this case, spatial studies combining multiple species are also similar to GSDRs, contributing to the confusion among patterns. Recently, CCSRs have also been constructed for bird [59] and phytoplankton [60] communities across space, and for a mammal community through time [51].

In general, CCSRs tend to be well described by power laws, $n_{\text {tot }} \propto{\overline{m_{\text {ind }}}}^{-b}$ (Box 2). The exponent, $b$, tends to be similar to the inverse of the scaling exponent of metabolic rates in the organisms studied [15,51,59,60] or to their geometric size [56]. In addition to observational data, there is also experimental support for power law CCSRs. Long and colleagues [61] have shown that a variety of communities composed of bacteria, algae and protozoa, inoculated with different sizes and numbers of species, all converge to fall close to a single inverse relationship ( $b=1)$ between mass and total community density (Figure 1d). The measured respiration of the 
communities is invariant with respect to average size, suggesting that energetic equivalence across communities is occurring in this study.

Regular patterns in CCSRs across a wide array of systems and taxa suggest a similar and powerful constraint operating across these different systems. Where resource availability or space is constant across sites or through time, the average amount of resource (or space) used per individual directly determines the number of individuals that can be supported [62]. In other words, power law CCSRs are expected to result simply from basic energy balance arguments with zero-sum competition for limiting resources or space ([15,51]; although the averaging shown above is typically not correct for these models, Box 2). If correct, then the CCSR is fairly straightforward to understand mechanistically, compared with other size-abundance relationships. If the limiting resource for the taxonomic group being studied is understood, the relationship between size and resource requirements has been quantified and any covariance between size and resource availability is known then it should be possible to predict the functional form of the CCSR [15,51,56,59].

\section{Towards a synthetic understanding of size-abundance relationships}

The existence of multiple relationships between size and abundance suggests the need for a synthetic understanding that acknowledges differences between the relationships while seeking to understand the linkages between them. The first step is to recognize that these relationships are distinct and that different combinations of underlying processes are responsible for each pattern (Glossary; Box 1). Specifically, the LSDR reflects processes influencing resource allocation among species, the ISD results from processes governing the distribution of individual sizes, the CCSR is generated by general constraints, such as resource limitation, on the 
community as a whole, and the GSDR reflects the operation of ecological and evolutionary processes at large spatiotemporal scales. The confusion among these patterns probably stems from an intuitive understanding that they must be related in meaningful ways. Although different measures of size and abundance are used in each relationship, they are still all measures of size and abundance. It is therefore necessary to understand not only the individual patterns but also the linkages among them.

From a technical perspective, linkages among these patterns are, at least for determinant growers, relatively straightforward (Figure 2). Linking LSDRs to ISDs requires knowledge of the distribution of body sizes of species (the species size distribution; Figure 2). Because the total number of individuals in a size class, $n_{i}$, is equal to the number of species in that size class $\left(S_{i}\right)$ multiplied by the average population density per species in that size class $\left(\bar{N}_{i}^{S}\right)$, the ISD and the LSDR are mathematically linked: $n_{i}=S_{i} \bar{N}_{i}^{S}[30,63,64]$. This link between the size distribution and the LSDR is important because it highlights the fact that one of the patterns might be generated by the other in combination with the species size distribution. For example: could the LSDR exhibit exponents significantly shallower than -0.75 because it is generated not directly by resource division among species but through the effects of resource division among size classes (characterized by the ISD) and the number of species in each size class? Understanding which patterns are mechanistically driven and which are simply combinations of other patterns will be critical to a general understanding of size-abundance relationships. For indeterminant growers, and other cases where a single species can be distributed across many body-size classes, this simple approach is insufficient, and work on more complicated situations is needed (e.g., Refs $[11,65])$. 
It is also possible to link the CCSR to the other size-density relationships because the mass values used in CCSRs are averages across each ISD (Figure 2). Understanding the CCSR is directly related to understanding how the shape of the ISD changes as a function of the number of individuals present in the community and their range of sizes. To our knowledge, only simple characteristics of the size distribution (such as average size) have been studied in this manner (e.g. Ref [66]). However, a more general understanding could be reached by studying shifts in the shape of the entire size distribution as a function of ecological and environmental factors.

The ability to link individual patterns suggests productive avenues for future research. It may also prove fruitful to recognize the considerable diversity of observed patterns for each type of relationship. For instance, there are notable differences in size-abundance relationships among different taxonomic groups and environments (Box 4). Although differences have generally been interpreted as challenging the generality of a given pattern, they also provide important insights into these relationships by highlighting the aspects of life history that influence how communities are structured. Comparing trophic and life-history differences should help isolate the important processes that affect the shape of these relationships (Box 4).

For decades, the relationship between size and abundance has been an important area of research in ecology. However, a general understanding of how size and abundance are related is still lacking as we have consensus on neither the general shapes of the patterns, nor the mechanisms underlying them. We suggest that these issues stem in part from confusion among the different patterns being analyzed (see also [11]), and also from meaningful differences between taxonomic and functional groups. The existence of these separate, but related, patterns and the insights to be gained from trophic relations and life history provides an ideal opportunity to produce a general understanding of the relationships between body size and abundance. Work 
addressing the linkages between patterns, both statistically and mechanistically, and the different behaviors of different groups should lead the way in our attempt for broader understanding.

\section{Acknowledgements}

We are indebted to John Damuth for his substantial influence on our thinking about the relationship between global and local size-density relationships. He, as well as Zach Long, Brian McGill, Sabrina Russo and an anonymous reviewer provided valuable comments on an early version of this manuscript. We thank Andrew Allen, Zach Long, Peter Morin, Scott Robinson, Sabrina Russo, and John Terborgh for kindly providing data for our figures. We also used data from the Forest Dynamics Plot of Barro Colorado Island (part of the Center for Tropical Forest Science), which were made possible through the support of NSF, the MacArthur Foundation, and the Smithsonian Tropical Research Institute and through the hard work of over 100 people. Data collected by J. Halfpenny were provided by the NSF supported Niwot Ridge LTER and the University of Colorado Mountain Research Station. E.P.W. was supported by an NSF Postdoctoral Fellowship in Biological Informatics (DBI-0532847). Authorship is in order of contribution. 


\section{References}

1 Peters, R.H. (1983) The ecological implications of body size, Cambridge University Press

2 Brown, J.H. et al. (2004) Toward a metabolic theory of ecology. Ecology 85, 1771-1789

3 Kerr, S.R. and Dickie, L.M. (2001) Biomass Spectrum, Columbia University Press

4 Abele, L.G. (1976) Comparative Species Richness in Fluctuating and Constant Environments:

Coral-Associated Decapod Crustaceans. Science 192, 461-463

5 Woodward, G. et al. (2005) Body size in ecological networks. Trends Ecol Evol 20, 402

6 Brown, J.H. (1995) Macroecology, The University of Chicago Press

7 Gaston, K.J. and Blackburn, T. (2000) Pattern and Process in Macroecology, Blackwell Science

8 Blackburn, T.M. et al. (1993) The relationship between abundance and body-size in natural animal assemblages. J Anim Ecol 62, 519-528

9 Loeuille, N. and Loreau, M. (2006) Evolution of body size in food webs: does the energetic equivalence rule hold? Ecol Lett 9, 171-178

10 Cotgreave, P. (1993) The Relationship Between Body-Size And Population Abundance In Animals. Trends Ecol Evol 8, 244-248

11 Jennings, S. et al. (2007) Measurement of body size and abundance in tests of macroecological and food web theory. J Anim Ecol 76, 72-82

12 Allen, A.P. et al. (2002) Global biodiversity, biochemical kinetics, and the energeticequivalence rule. Science 297, 1545-1548

13 Damuth, J. (1987) Interspecific allometry of population-density in mammals and other animals - the independence of body-mass and population energy-use. Biol J Linn Soc 31, 193246 
14 Cyr, H. et al. (1997) Population density and community size structure: comparison of aquatic and terrestrial systems. Oikos 80, 139-149

15 Enquist, B.J. et al. (1998) Allometric scaling of plant energetics and population density.

Nature 395, 163-165

16 Marquet, P.A. et al. (1995) Body-size, population-density, and the energetic equivalence rule. J Anim Ecol 64, 325-332

17 Lawton, J.H. (1990) Species Richness And Population-Dynamics Of Animal Assemblages Patterns In Body Size - Abundance Space. Phil. Trans. R. Soc. B 330, 283-291

18 Damuth, J. (1981) Population-density and body size in mammals. Nature 290, 699-700 19 Marquet, P.A. (2002) Of predators, prey, and power laws. Science 295, 2229-2230 20 Ernest, S.K.M. et al. (2003) Thermodynamic and metabolic effects on the scaling of production and population energy use. Ecol Lett 6, 990-995

21 Meehan, T.D. (2006) Energy use and animal abundance in litter and soil communities. Ecology 87, 1650-1658

22 Carbone, C. and Gittleman, J.L. (2002) A common rule for the scaling of carnivore density. Science 295, 2273-2276

23 Marquet, P.A. et al. (1990) Scaling Population-Density to Body Size in Rocky Intertidal Communities. Science 250, 1125-1127

24 Blackburn, T.M. and Gaston, K.J. (1999) The relationship between animal abundance and body size: a review of the mechanisms. Adv Ecol Res 28, 181-210

25 Van Valen, L.M. (1983) How pervasive is coevolution? In Coevolution (Nitecki, M.H., ed.), pp. 1-19, University of Chicago Press 
26 Damuth, J. (2007) A macroevolutionary explanation for energy equivalence in the scaling of body size and population density. Am Nat in press

27 Van Valen, L.M. (1973) A new evolutionary law. Evolutionary Theory 1, 1-30

28 Blackburn, T.M. and Gaston, K.J. (1997) A critical assessment of the form of the interspecific relationship between abundance and body size in animals. J Anim Ecol 66, 233-249

29 Schmid, P.E. et al. (2002) Scaling in stream communities. Proc. R. Soc. B 269, 2587-2594

30 Jonsson, T. et al. (2005) Food webs, body size, and species abundance in ecological community description. In Advances In Ecological Research, Vol 36, pp. 1-84

31 Cyr, H. (2000) Individual energy use and the allometry of population density. In Scaling in Biology (Brown, J.H. and West, G.B., eds), pp. 267-283, Oxford University Press

32 Russo, S.E. et al. (2003) Size-abundance relationships in an Amazonian bird community: Implications for the energetic equivalence rule. Am Nat 161, 267-283

33 Nee, S. et al. (1991) The Relationship Between Abundance And Body Size In British Birds. Nature 351, 312-313

34 Cotgreave, P. (1994) The relation between body-size and abundance in a bird community the effects of phylogeny and competition. Proc. R. Soc. B 256, 147-149

35 Holling, C.S. (1992) Cross-scale morphology, geometry, and dynamics of ecosystems. Ecol Monogr 62, 447-502

36 Ernest, S.K.M. (2005) Body size, energy use, and community structure of small mammals. Ecology 86, 1407-1413

37 Brown, J.H. et al. (1995) Spatial variation in abundance. Ecology 76, 2028-2043

38 Murphy, H.T. et al. (2006) Distribution of abundance across the range in eastern North American trees. Glob Ecol Biogeogr 15, 63-71 
39 Currie, D.J. (1993) What shape is the relationship between body size and population-density. Oikos 66, 353-358

40 Lawton, J.H. (1989) What is the relationship between population-density and body size in animals. Oikos 55, 429-434

41 Siemann, E. et al. (1996) Insect species diversity, abundance and body size relationships. Nature 380, 704-706

42 McClain, C.R. (2004) Connecting species richness, abundance and body size in deep-sea gastropods. Glob Ecol Biogeogr 13, 327-334

43 Sheldon, R.W. et al. (1972) The size distribution of particles in the ocean. Limnol Oceanogr $17,327-340$

44 Griffiths, D. (1986) Size-abundance relations in communities. Am Nat 127, 140-166 45 Irwin, A.J. et al. (2006) Scaling-up from nutrient physiology to the size-structure of phytoplankton communities. J Plankton Res 28, 459-471

46 Muller-Landau, H.C. et al. (2006) Comparing tropical forest tree size distributions with the predictions of metabolic ecology and equilibrium models. Ecol Lett 9, 589-602

47 Meyer, H.A. et al. (1961) Forest Management, The Ronald Press Company 48 Enquist, B.J. and Niklas, K.J. (2001) Invariant scaling relations across tree-dominated communities. Nature 410, 655-660

49 Coomes, D.A. et al. (2003) Disturbances prevent stem size-density distributions in natural forests from following scaling relationships. Ecol Lett 6, 980-989

50 Morse, D.R. et al. (1985) Fractal dimension of vegetation and the distribution of arthropod body lengths. Nature 314, 731-733 
51 White, E.P. et al. (2004) Trade-offs in community properties through time in a desert rodent community. Am Nat 164, 670-676

52 Carbone, C. et al. (1999) Energetic constraints on the diet of terrestrial carnivores. Nature 402, 286-288

53 Muller-Landau, H.C. et al. (2006) Testing metabolic ecology theory for allometric scaling of tree size, growth and mortality in tropical forests. Ecol Lett 9, 575-588

54 Coomes, D.A. (2006) Challenges to the generality of the WBE theory. Trends Ecol Evol 11, 593-596

55 Ritchie, M.E. and Olff, H. (1999) Spatial scaling laws yield a synthetic theory of biodiversity. Nature 400, 557-560

56 Yoda, K. et al. (1963) Self-thinning in overcrowded pure stands under cultivated and natural conditions (Intraspecific competition among higher plants XI). J. Inst. Polytech. Osaka City Uni. Ser. D 14, 107-129

57 Westoby, M. (1984) The self-thinning rule. Adv Ecol Res 14, 167-225

58 Hughes, R.N. and Griffiths, C.L. (1988) Self-Thinning In Barnacles And Mussels - The Geometry Of Packing. Am Nat 132, 484-491

59 Meehan, T.D. et al. (2004) Energetic determinants of abundance in winter landbird communities. Ecol Lett 7, 532-537

60 Li, W.K.W. (2002) Macroecological patterns of phytoplankton in the northwestern North Atlantic Ocean. Nature 419, 154-157

61 Long, Z.T. and Morin, P.J. (2005) Effects of organism size and community composition on ecosystem functioning. Ecol Lett 8, 1271-1282 
62 Ernest, S.K.M. and Brown, J.H. (2001) Homeostasis and compensation: The role of species and resources in ecosystem stability. Ecology 82, 2118-2132

63 Harvey, P.H. and Lawton, J.H. (1986) Community ecology: patterns in 3 dimensions. Nature 324, 212-212

64 Damuth, J. (1994) No conflict among abundance rules. Trends Ecol Evol 9, 487-487

65 Rinaldo, A. et al. (2002) Cross-scale ecological dynamics and microbial size spectra in marine ecosystems. Proc. R. Soc. B 269, 2051-2059

66 Kaspari, M. (2005) Global energy gradients and size incolonial organisms: Worker mass and worker number in ant colonies. Proc Natl Acad Sci U S A 102, 5079-5083

67 Griffiths, D. (1992) Size, abundance, and energy use in communities. J Anim Ecol 61, 307315

68 Kerkhoff, A.J. and Enquist, B.J. (2006) Ecosystem allometry: the scaling of nutrient stocks and primary productivity across plant communities. Ecol Lett 9, 419-427

69 Weitz, J.S. and Levin, S.A. (2006) Size and scaling of predator-prey dynamics. Ecol Lett 9, 548-557

70 Loehle, C. (2006) Species abundance distributions result from body size-energetics relationships. Ecology 87, 2221-2226

71 Ackerman, J.L. et al. (2004) The contribution of small individuals to density-body size relationships: examination of energetic equivalence in reef fishes. Oecologia 139, 568-571 72 Silverman, B.W. (1986) Density estimation for statistics and data analysis, Chapman \& Hall/CRC 
73 Clark, R.M. et al. (1999) Generalizations of power-law distributions applicable to sampled fault-trace lengths: model choice, parameter estimation and caveats. Geophysical Journal International 136, 357-372

74 Savage, V.M. (2004) Improved approximations to scaling relationships for species, populations, and ecosystems across latitudinal and elevational gradients. J Theor Biol 227, 525534

75 Condit, R. (1998) Tropical Forest Census Plots, Springer-Verlag and R.G. Landes Co. 


\section{Glossary}

Cross-community scaling relationship ${ }^{*}$ (CCSR): describes the relationship between the average size of an individual in an assemblage $\left(\bar{m}_{\text {Ind }}\right.$; or the average of some transformation of size; Box 2) and the total number of individuals in that assemblage $\left(n_{\text {Tot }}\right)$. Each point is from a spatially or temporally distinct community. The most common form of the CCS is the self-thinning rule observed in plant and invertebrate populations.

Damuth's rule: a special case of the GSDR, in which the relationship is characterized by a power law with an exponent of $-3 / 4$.

Energetic equivalence rule (EER): a special case of the GSDR (and sometimes the LSDR) in which the relationship between body size and density is the inverse of the relationship between body size and metabolic rate. The exponent need not necessarily be equal to $-3 / 4$ if the scaling of metabolic rate deviates from this exponent (e.g., Ref [32]).

Energetic equivalence: a more general concept than the EER, in which for any of the four relationships between size and abundance, the change in abundance is compensated for by the change in metabolic rate such that populations, or size classes, or communities (whatever the relevant measure) exhibit an invariance in energy use with respect to size.

Global size-density relationship ${ }^{*}$ (GSDR): The relationship between the average body size of a species $\left(M_{s p}\right)$ and its average population density compiled from the literature $\left(N_{\text {comp }}\right)$. Densities may be taken from any point on the globe. This is the pattern originally plotted by Damuth [18]. 
Local size-density relationship* (LSDR): the relationship between the average body size of a species $(\bar{M})$ and its population density $(N)$ where all population densities are taken from a single region.

Individual size distribution $^{*}$ (SD) or size spectrum: these terms (the first traditionally terrestrial and the second traditionally aquatic) describe the pattern relating the number of individuals in a body-size class (irrespective of species; $n_{i}$ ) and the average size of that body size class $\left(\bar{m}_{i}\right)$. More generally, this is a frequency distribution (or probability density) of body sizes of individuals in a community. We recommend the use of the term size distribution because it is more descriptive.

* One of the four relationships between size and abundance described in the text. 


\section{Box 1. Confusion among different size-abundance relationships}

The existence of four different relationships between size and abundance has created substantial confusion in the literature [11]. It is not uncommon to see (i) the SDR and ISD discussed as if they were the same pattern (e.g. Refs [45,67]), (ii) to see CCSR relationships described as validating the EER form of the GSDR (e.g. Refs [60,61]), and (iii) to see predictions regarding the size distribution made using models for CCSRs (e.g. Refs [48,68]). One recent paper mixes together the size-distribution and the CCSR by randomly choosing individual body size bins from across geographically separate ISDs, further clouding the distinctions among patterns [21].

From a quantitative viewpoint the four relationships are very different, neither the abundance measures $\left(N_{\text {comp }}, N, n_{i}, n_{\text {tot }}\right)$ nor usually the measures of size $\left(M_{s p}, M_{s p}, \bar{m}_{i}, \overline{m_{\text {ind }}}\right)$ are equivalent across patterns. Because these are all measures of size and abundance, it is intuitive to think of these patterns as related. However, confounding these relationships without explicitly reconciling the different measures being used is confusing and undesirable.

Even when differences between the patterns are recognized, these differences are sometimes discussed in terms of which approach to analyzing the data is best $[2,11]$. However, the different patterns simply characterize different ways of looking at a system, and while a given pattern may be best for addressing a particular question there is no single best way to analyze this type of data.

This confusion among patterns can have profound impacts when modeling abundance in local communities as a function of size. Many community models assume that the LSDR will be of the form $N \propto M_{s p}^{-3 / 4}$ (e.g., [12,69,70]), typically justifying this assumption using Damuth’s Rule. However, size is generally a poor predictor of abundance in most local communities 
regardless of which hypothesized mechanism generates the observed differences between the LSDR and GSDR. In addition, if a community model actually relies on the total abundance in a size class - as might be the case in predator-prey or plant community models - then the sizedistribution would be the more appropriate relationship [11,68], and depending on the taxonomic group simple generalizations may or may not be justified. 


\section{Box 2. Statistical issues}

\section{Size Density Relationships:}

Regression method: A variety of regression methods have been used to fit SDRs (e.g., OLS and RMA). These approaches typically produce different results [29,67], and plausible arguments have been made for using many of these methods [16]. This produces challenges for interpreting whether a SDR exhibits a particular slope [8,29,67]. In addition, several studies have used phylogenetically independent contrasts to evaluate the relationship [7,32], but it is unclear whether abundance is actually a phylogenetically conserved trait.

Artifacts: Several potential artifacts exist in the analysis of SDRs including (i) potential bias towards maximum abundances in literature compilations [16,17,40]; (ii) effects of census area on estimated densities that could exaggerate the correlation between size and abundance [7]; and (iii) the possibility for correlations between species richness and body size to generate constraint triangles in the absence of meaningful constraints $[7,39]$. To our knowledge thorough analyses of the influence of these artifacts on observed patterns have not been conducted [39].

What is a species' size: For determinant growers, average adult size is typically considered the appropriate measure of species size. For indeterminant growers, a species size is much less straightforward. This presents a substantial problem for constructing SDRs for indeterminant growers [71]. Methods for characterizing species’ size in these taxa are now being discussed in the literature [11]. 
Size-distribution: A number of approaches have been used to characterize the size-distribution including: linear binning [48], logarithmic binning [41], normalized-logarithmic binning [3], maximum-likelihood based model competition [46], and fitting the cumulative distribution function [65]. Binning based approaches are useful for looking at the form of the distribution, but are sensitive to both the bin width and the location of bin edges [72] which may cause problems for parameter estimation using regressions on binned data. Cumulative distribution and maximum likelihood based approaches can only be used when a particular model has been specified, in which case maximum likelihood methods are generally considered superior [73].

Cross Community Scaling: Predicted relationships in CCSR studies are based on the idea that the number of individuals in an assemblage should be equal to the resource utilized by that assemblage divided by the average resource use per individual. Average resource use is typically estimated using a non-linear functions relating size and resource use. However, the average value of a non-linear function is not the same as the value of that non-linear function evaluated at the average [74]. The vast majority of CCSR studies ignore this issue, plotting total abundance as a function of average mass. While this approximation may be reasonable in some instances it is more appropriate to plot abundance as a function of average estimated resource use (e.g., $\overline{m_{\text {ind }}^{3 / 4}}$ not $\overline{m_{\text {ind }}}{ }^{3 / 4}$ ). 


\section{Box 3. Alternative currencies}

\section{Alternative currencies for size:}

Most size-abundance studies define size as mass. However, size can also be measured using other metrics including length and volume. As long as the metric is directly proportional to mass (e.g. volume in most cases) this choice will have little effect on the observed relationship. However, using currencies that are not directly proportional to mass can change the observed relationship and care should be taken when comparing patterns based on different measures of body size.

\section{Alternative currencies for abundance:}

Although we focus on the relationship between body size and abundance, it is also common to consider the relationship between body size and other currencies such as biomass, energy flux and nutrients $[3,36,68]$. Evaluating currencies other than abundance is important especially for questions related to resource partitioning. Because of size-based differences in resource use, sizeabundance distributions can give different impressions of dominance and importance relative to size distributions using alternative size-weighted currencies (e.g. biomass or energy flux) [36]. In addition, general behavior might reveal itself in some currencies but not others, suggesting avenues for understanding general patterns [24].

Alternative currencies can often be measured directly or estimated from their relationship with the mass of an individual. For example, the population energy use $\left(B_{\text {tot }}\right)$ of a species can be estimated by multiplying its population density $\left(N_{\text {comp }}\right)$ by its estimated metabolic rate based on mass $\left(M_{s p}\right)$ and the appropriate normalization constant $\left(b_{0}\right)$, such 
that $B_{\text {tot }}=N_{\text {comp }} B_{\text {indiv }}=N_{\text {comp }} b_{0} M_{s p}^{3 / 4}$. This was the logic used to argue for the EER $[18,20]$. It is important to remember that error propagation might affect the validity of the estimated values. Aquatic ecologists studying ISDs also use alternative currencies, typically plotting total biomass in a size class instead of abundance [3], and energy flux has also been used [36]. One challenge for working with alternative currencies when studying size distributions is that using a measure other than abundance makes it impossible to use traditional statistical methods associated with the analysis of probability distributions [36].

The fact that different currencies can result in different patterns is advantageous for understanding ecological systems. However, these differences can also result in confusion when comparing the results of different studies. For example, aquatic size distributions are often examined using biomass, while terrestrial studies tend to use abundance. Therefore the form of these relationships cannot be directly compared to one another without taking this difference into account. 


\section{Box 4. Future directions: synthesizing the diversity of relationships}

Within the numerous size-abundance relationships, there appear to be both similarities of form within groups and regular differences between groups. An exciting avenue for future research will be to understand what processes create these seemingly regular similarities and differences in size-abundance relationships across groups of organisms or ecosystems. Possible avenues for grouping patterns that show promise for highlighting important processes include:

- Trophic differences. Trophic differences are important for understanding both individual size distributions and global and local size-density relationships. Most of the work in this area has focused on the effects of decreasing energy availability with trophic level [1921], however, even within a trophic level, there appear to be important differences between trophic guilds in their size-abundance relationships [32]. Understanding why trophic levels and guilds differ could provide insights into underlying differences in how size is related to access to resources

- Life-history differences. Organisms with indeterminant growth, little parental care and a sessile (or passively dispersed) life history appear to exhibit fairly regular patterns in both the LSDR and size spectra (e.g. Refs [3,31]). Whereas organisms with determinant growth, significant parental care and highly directed mobility tend to exhibit less regular local-scale patterns (e.g. Refs [7,36]). There are reasons to suspect that life-history differences drive differences between groups. For example, sessile and mobile organisms have different constraints on how resources can be partitioned. Mobile organisms partition resources more flexibly in space and time (via internal and external resource storage, territoriality and migration) whereas sessile organisms are restricted to 
sequestering resources via growth and the pre-emption of space. Similar arguments can be made for the effects of other life history characteristics (e.g. parental care) on resource partitioning and competition along a body-size axis (see discussion in ISD section).

- Phylogenetic relatedness. Relationships restricted to closely related species (i.e. species within a genus) often show different patterns from those containing a phylogenetically diverse array of species. For example, Nee et al. [33] have shown positive LSDRs within a genus as opposed to the more common negative relationship including all genera [33]. Changes in the pattern at different levels of taxonomy, or phylogeny, have important implications for inferring structuring processes. In this case the positive relationship within genera may reflect the competitive advantage of larger species over closely related smaller species, suggesting that the mechanism for the observed decrease in abundance occurs at a higher taxonomic level. 


\section{Figure Legends}

Figure 1. The four relationships between body size and abundance. (a) Global size-density relationship plot combining mammals (red circles) and assorted ectotherms (blue squares). Data are plotted as abundance at $37^{\circ} \mathrm{C}$ using methods similar to those described in Ref [12], but using a multiple regression to determine the temperature correction. The relationship is strong, $\mathrm{r}^{2}=$ 0.80 , and the exponent is close to $-0.75, N_{\text {comp }} \propto M_{s p}^{-.746}$. (b) Local size-density relationship plot for birds in Manu National Park in Peru. Species are separated into trophic groups - frugivoreomnivore (red squares), granivores (black point up triangles), insectivores (blue circles), and raptors (green point down triangles). The relationship (combining all trophic groups) is weak $\left(\mathrm{r}^{2}\right.$ $=0.08$ ) and has an exponent equal to -0.22 , substantially less negative than -0.75 . Regression lines for trophic groups differ from the overall relationship and from each other. (c) Individual size distributions for mammals at Niwot Ridge (red; units are grams) and trees at Barro Colorado Island (blue; units are kg). Tree data were converted from diameter to mass using the equation in [48]. Both distributions are presented using the method described in Ref [3]. The tree distribution exhibits a simply monotonic decline, whereas the mammal distribution is more complex and multi-modal. (d) Experimental cross-community scaling relationship for communities composed of bacteria, algae, and protozoa. Communities inoculated with different combinations of high richness (triangles), low richness (squares), large species (blue), and small species (red) all converge to approximately the same relationship (exponent $=-1.02 ; r^{2}=0.89$ ). Data from Refs [12,13] (a), Ref [32] (b), http://culter.colorado.edu/NWT, http://ctfs.si.edu/datasets/bci (we followed similar data processing protocols to those used in [46]) and Refs [36,75] (c) and Ref [61] (d). 
Figure 2. Linkages between the four size-abundance relationships. The local size density relationship [LSDR; (a)] is related to the global size density relationship [GSDR; (b,c)] in one of two ways. The LSDR is either a simple subset of the GSDR points [open circles indicate species not observed in the local sample, black circles indicate species that are; (b)] or, alternatively, only the most abundant species from an LSDR (red circles) occur in the GSDR (c). LSDRs are linked to individual size distributions [ISDs; (d)] by summing the abundances of all of the species in a body size bin and plotting that value against the average mass of that bin (blue squares; this linkage is more complicated if species can occur in $>1$ size class). Further summing the abundances across all body size bins and plotting this against the average body size of an individual in the community produces a single point in a cross-community scaling relationship [CCSR; green square; (d)]. 

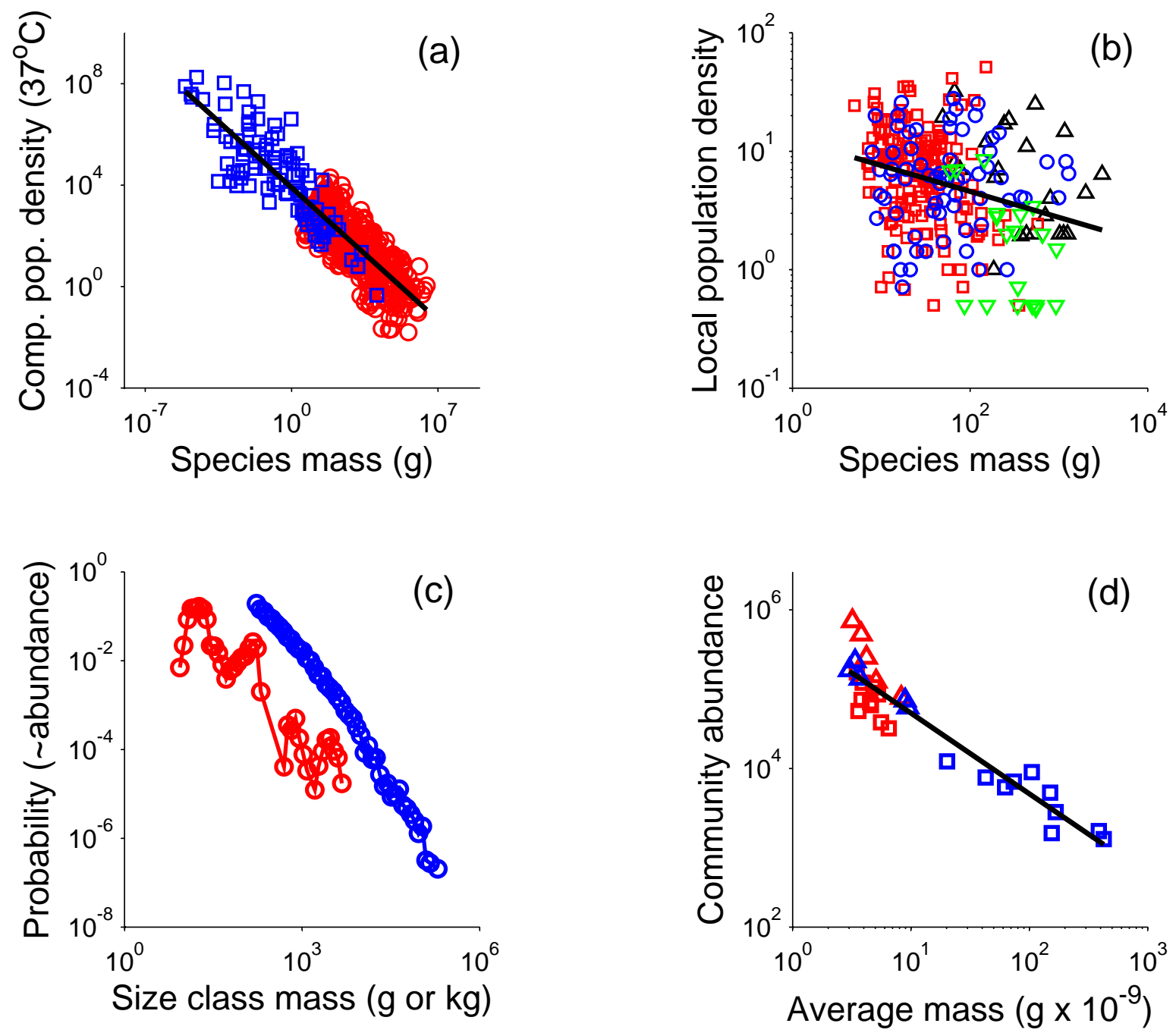


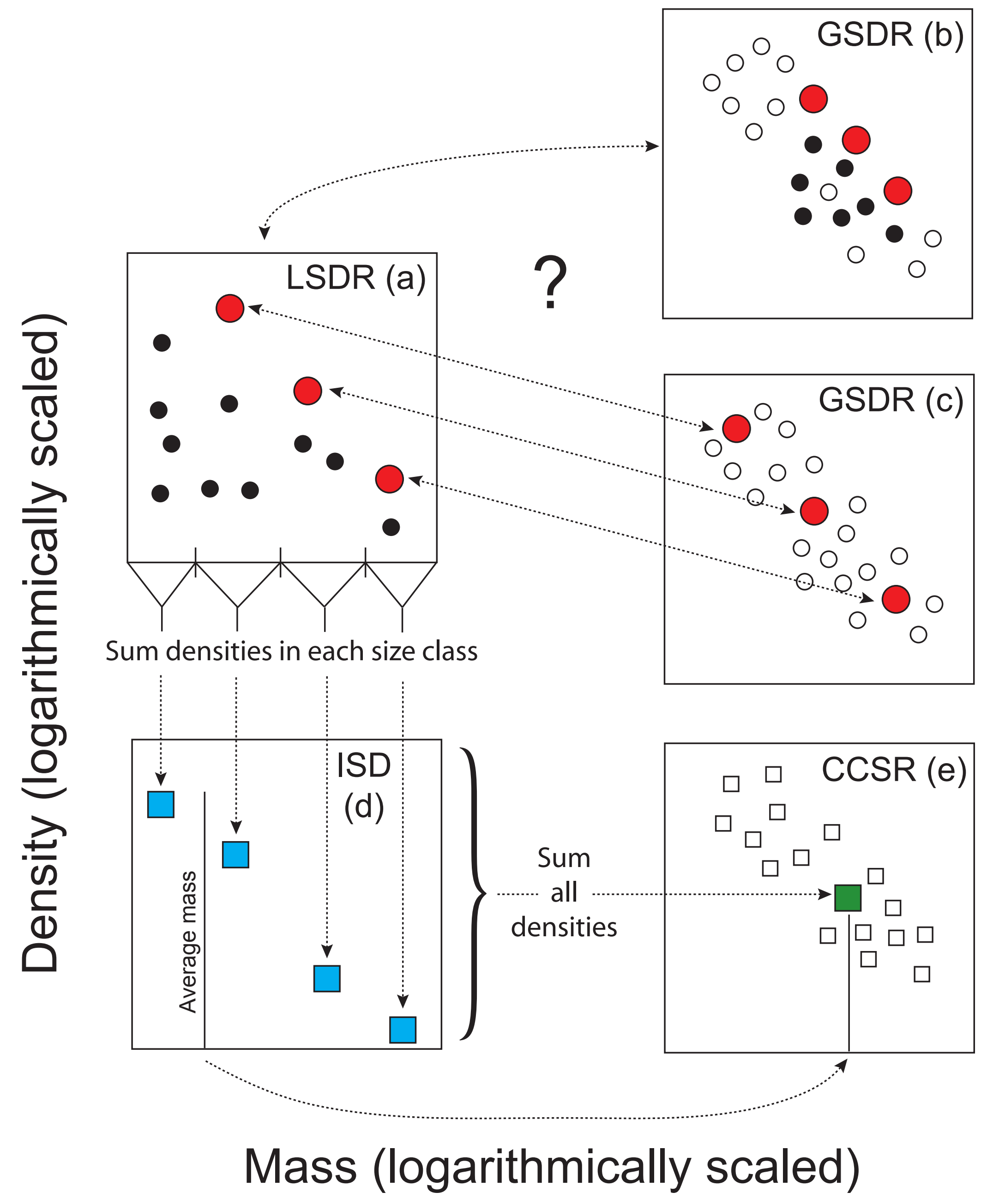

